The Journal of $\mathbf{N}_{\text {onlinear }} \mathbf{S}_{\text {ciences and }}$ A pplications

http://www.tjnsa.com

\title{
T-ROUGH SEMIPRIME IDEALS ON COMMUTATIVE RINGS
}

\author{
S. B. HOSSEINI ${ }^{1 *}$
}

Communicated by S. Jafari

\begin{abstract}
Rough sets were originally proposed in the presence of an equivalence relation. An equivalence relation is sometimes difficult to be obtained in rearward problems due to the vagueness and incompleteness of human knowledge. The purpose of this paper is to introduce and discuss the concept of $T$-rough semiprime ideal, $T$-rough fuzzy semiprime ideal and $T$-rough quotient ideal in a commutative ring which are a generalization of rough set and approximation theory. We compare relation between a rough ideal and a $T$-rough ideal and prove some theorems.
\end{abstract}

\section{INTRODUCTION}

The notion of rough sets has been introduced by Z. Pawlak [16-23] and Z. Pawlak, A. Skowron [24-26]. It soon invoked a natural question concerning possible connection between rough sets and algebraic systems. The algebraic approach to rough sets have been given and studied by T. Iwinski [11], A. Rosenfeild [27], W. Zhang, W. Wu [31]. R. Biswas [1, 2], R. Biswas, S. Nanda [3] introduced the notion of rough set and rough subgroups. N. Kuroki [14] introduced the notion of rough ideals in a semigroups. B. Davvaz [6] introduced the notion of rough subring with respect to an ideal of a ring. D. Dubois, H. Prade [7, 8] combined fuzzy sets and rough sets in a fruitful way by defining rough fuzzy sets and fuzzy rough sets. Qi-Mei Xiao, Zhen-Liang Zhang [28] discussed the lower and the upper approximations of prime ideals and fuzzy prime ideals in a semigroup with details.

Date: Received: August 30, 2011; Revised: November 16, 2011.

${ }^{*}$ Corresponding author

(c) 2011 N.A.G.

2000 Mathematics Subject Classification. 03B52, 03F55, $06 \mathrm{D} 72$.

Key words and phrases. approximation space; rough ideal; semiprime ideal; T-rough set; set-valued homomorphism; $T$-rough semiprime ideal; $T$-rough fuzzy ideal; commutative ring. 
B. Davvaz [5] introduced $T$-rough set and $T$-rough homomorphism in a group. Rough set theory is an extension of set theory, in which a subset of a universe is described by a pair of ordinary sets called the lower and upper approximations. The lower approximation of a given set is the union of all the equivalence classes which are subsets of the set, and the upper approximation is the union of all the equivalence classes which have a non-empty intersection with the set. It is well known that a partition induces an equivalence relation on a set and vice versa. The properties of rough sets can thus be examined via either partition or equivalence classes. Rough sets are a suitable mathematical model of vague concepts, i.e., concepts without sharp boundaries. S. B. Hosseini et al. $[9,10]$ studied some properties of $T$-rough set in semigroup and commutative rings. In this paper, we discussed $T$-rough ideal, $T$-rough semiprime ideal, $T$-rough fuzzy ideal and $T$-rough fuzzy semiprime ideal of rings based on definitions in [5] and generalized some theorems have been proved [5, 6, 10, 28, 29].

\section{Preliminaries}

The following definitions and preliminaries are required in the sequel of the work and hence presented in brief. Some of them were in [6]. Suppose that $U$ is a non-empty set. A partition or classification of $U$ is a family $\Theta$ of non-empty subsets of $U$ such that each element of $U$ is contained in exactly one element of $\Theta$. It is vitally important to recall that an equivalence relation $\theta$ on a set $U$ is a reflexive, symmetric and transitive binary relation on $U$. Each partition $\Theta$ induces an equivalence relation $\theta$ on $U$ by setting

$$
x \theta y \Leftrightarrow x \text { and } y \text { are in the same class of } \Theta \text {. }
$$

Conversely, each equivalence relation $\theta$ on $U$ induces a partition $\Theta$ of $U$ whose classes have the form

$$
[x]_{\theta}=\{y \in U \mid x \theta y\} .
$$

Definition 2.1. A pair $(U, \theta)$ where $U \neq \emptyset$ and $\theta$ is an equivalence relation on $U$ is called an approximation space.

Definition 2.2. For an approximation space $(U, \theta)$ by a rough approximation in $(U, \theta)$ we mean a mapping $A p r: P(U) \longrightarrow P(U) \times P(U)$ defined by for every $X \in P(U), \operatorname{Apr}(X)=(\operatorname{Apr}(X), \overline{A p r}(X))$, where

$$
\underline{\operatorname{Apr}}(X)=\left\{x \in U \mid[x]_{\theta} \subseteq X\right\}, \overline{A p r}(X)=\left\{x \in U \mid[x]_{\theta} \bigcap X \neq \emptyset\right\} .
$$

$\operatorname{Apr}(X)$ is called the lower rough approximation of $X$ in $(U, \theta)$ whereas $\overline{A p r}(X)$ is called the upper rough approximation of $X$ in $(U, \theta)$.

Definition 2.3. [16] Given an approximation space $(U, \theta)$ a pair $(A, B)$ in $P(U) \times$ $P(U)$ is called a rough set in $(U, \theta)$ if $(A, B)=(\underline{A p r}(X), \overline{A p r}(X))$ for some $X \in P(U)$.

Definition 2.4. A subset $X$ of $U$ is called definable if $\operatorname{Apr}(X)=\overline{A p r}(X)$. If $X \subseteq U$ is given by a predicate $P$ and $x \in U$, then

(1) $x \in \underline{A p r}(X)$ means that $x$ certainly has property $P$, 
(2) $x \in \overline{\operatorname{Apr}}(X)$ means that $x$ possibly has property $P$,

(3) $x \in U \backslash \overline{A p r}(X)$ means that $x$ definitely does not have property $P$.

The rough complement of $\operatorname{Apr}(A)$ denoted by $A \operatorname{pr}^{c}(A)$ is defined by

$$
\operatorname{Apr}^{c}(A)=(U \backslash \overline{\operatorname{Apr}}(A), U \backslash \underline{A p r}(A) \text {. }
$$

Also, we can define $\operatorname{Apr}(A) \backslash A \operatorname{pr}(B)$ as follows:

$$
\operatorname{Apr}(A) \backslash \operatorname{Apr}(B)=(\underline{A p r}(A) \backslash \overline{A p r}(B), \overline{A p r}(A) \backslash \underline{A p r}(B)) .
$$

Proposition 2.5. [16] Let $U$ be a nonempty set and $\theta$ an equivalence relation on $U$. For every subsets $A, B \subseteq U$, we have

(i) $\operatorname{Apr}(A) \subseteq A \subseteq \overline{A p r}(A)$;

(ii) $\overline{\text { If } A} \subseteq B$, then $\operatorname{Apr}(A) \subseteq A p r(A)$ and $\overline{\operatorname{Apr}}(A) \subseteq \overline{\operatorname{Apr}}(B)$;

(iii) $\operatorname{Apr}(A \cap B)=\overline{A p r}(A) \cap \overline{A p r}(B)$;

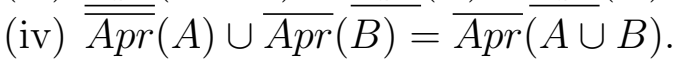

\section{T-Rough Ideal And $T$-Rough Semiprime Ideal in a COMmutative RING}

In this section, we define the concept of a set-valued homomorphism and give some important examples of set-valued mapping. We show that every ring homomorphism is a set-valued homomorphism. We also investigate some basic properties of the generalized lower and upper approximation operators in a ring. We generalize rough ideal [9] that is called $T$-rough ideal [3]. We apply the notion $T$-rough sets in a commutative ring .

Definition 3.1. $(i)$ Let $R$ be a ring and $I \neq \emptyset$ be a subset of $R$. $I$ is called an ideal of $R$, if $x r, r x, x-y \in I$ for all $r \in R$ and $x, y \in I$.

(ii) Let $U \neq \emptyset$ be a subset of $R$. $U$ is called a subring of $R$, if $x y, x-y \in U$ for all $x, y \in U$.

(iii) Let $A$ and $B$ are non-empty subsets of $R$. We denote $A B$ by the set of all finite sum $\left\{\sum_{i=1}^{n} a_{i} b_{i} \mid a_{i} \in A, b_{i} \in B, n \in \mathbf{N}\right\}$.

(iv) An ideal $P$ is called a prime ideal if $P \neq R$ and if $A, B \subseteq R$ be ideals of $R$ and $A B \subseteq P$, then $A \subseteq P$ or $B \subseteq P$. In a commutative ring $R$, primness is an equivalent with $x, y \in R$ and $x y \in P$ implies that $x \in P$ or $y \in P$.

(v) An ideal $Q \neq R$ is called a semiprime ideal of $R$, if $A$ be an ideal of $R$ and $A^{2} \subseteq Q$, then $A \subseteq Q$. In a commutative $\operatorname{ring} R$, semiprimness is an equivalent with $x \in R$ and $x^{2} \in Q$ implies that $x \in Q$.

It is clear that any prime ideal is a semiprime ideal but if $p_{1}, p_{2}$ be two prime numbers, then ideal $<p_{1} p_{2}>=p_{1} p_{2} \mathbf{Z}$ is a semiprime ideal of $\mathbf{Z}$ that is not prime.

Definition 3.2. [5] Let $X$ and $Y$ be two non-empty sets and $B \subseteq Y$. Let $T: X \rightarrow P^{*}(Y)$ be a set-valued mapping where $P^{*}(Y)$ denotes the set of all 
non-empty subsets of $Y$. The lower inverse and upper inverse of $B$ under $T$ are defined by

$$
T^{+}(B)=\{x \in X \mid T(x) \subseteq B\} ; T^{-1}(B)=\{x \in X \mid T(x) \cap B \neq \emptyset\} .
$$

Definition 3.3. [5] Let $X$ and $Y$ be two non-empty sets and $B \subseteq Y$. Let $T: X \rightarrow P^{*}(Y)$ be a set-valued mapping where $P^{*}(Y)$ denotes the set of all non-empty subsets of $Y .\left(T^{+}(B), T^{-1}(B)\right)$ is called $T$-rough set of $R$ with respect to $B$.

Example 3.4. $(i)$ Let $(U, \theta)$ be an approximation space and $T: U \rightarrow P^{*}(U)$ be a set-valued mapping where $T(x)=[x]_{\theta}$, then for any $B \subseteq U, T^{+}(B)=$ $\underline{A p r}(B)$ and $T^{-1}(B)=\overline{A p r}(B)$.

(ii) Let $\mathbf{N}$ be natural numbers set and $\mathbf{Z}$ be integer numbers set and $T$ : $\mathbf{N} \rightarrow P^{*}(\mathbf{Z})$ where for every $n \in \mathbf{N}, T(n)=\{1,2, \ldots, n\}$. If $B=\{1,2, \ldots, 10\}$, then $T^{+}(B)=\{1,2, \ldots, 10\}$ and $T^{-1}(B)=\mathbf{N}$.

(iii) Let $\mathbf{N}$ be natural numbers set and $\mathbf{Z}$ be integer numbers set and $T$ : $\mathbf{N} \rightarrow P^{*}(\mathbf{Z})$ where for every $n \in \mathbf{N}, T(n)=\{ \pm n\}$. If $B=\{1,2, \ldots, 10\}$, then $T^{+}(B)=\{1,2, \ldots, 10\}=T^{-1}(B)$.

(iv) Let $\mathbf{R}$ be real numbers set and $T: \mathbf{R} \rightarrow P^{*}(\mathbf{R})$ where for every $a \in \mathbf{R}, T(a)=$ $[-|a|,|a|]$. If $A=[0,1]$, then $T^{+}(A)=\{0\}$ and $T^{-1}(A)=[-1,1]$.

$(v)$ Let $\mathbf{Z}$ be integer numbers set and $T: \mathbf{Z} \rightarrow P^{*}(\mathbf{Z})$ where for every $n \in$ $\mathbf{Z}, T(n)=n \mathbf{Z}$. If $A=2 \mathbf{Z}$, then $T^{+}(A)=2 \mathbf{Z}$ and $T^{-1}(A)=\mathbf{Z}$.

(vi) Let $f: R \rightarrow S$ be a ring homomorphism and $A$ be a non-empty set $S$. Then for the set-valued mapping $T: R \rightarrow P^{*}(S)$ defined by $T(r)=\{f(r)\}$, we have $T^{-1}(A)=\{r \in R \mid f(r) \in A\}=T^{+}(A)=f^{-1}(A)$.

Proposition 3.5. [5] Let $X$ and $Y$ be two non-empty sets and $A, B \subseteq Y$. Let $T: X \rightarrow P^{*}(Y)$ be a set-valued mapping where $P^{*}(Y)$ denotes the set of all non-empty subsets of $Y$. Then the following hold:

(1) $T^{-1}(A \cup B)=T^{-1}(A) \cup T^{-1}(B)$;

(2) $T^{+}(A \cap B)=T^{+}(A) \cap T^{+}(B)$;

(3) $A \subseteq B$ implies $T^{+}(A) \subseteq T^{+}(B)$ and $T^{-1}(A) \subseteq T^{-1}(B)$;

(4) $T^{+}(A \cup B) \subseteq T^{+}(A) \cup T^{+}(B)$ and $T^{-1}(A \cap B) \subseteq T^{-1}(A) \cap T^{-1}(B)$.

Using the lower inverse and upper inverse under $T$, we define a binary relation on subsets of $Y$ as follows:

$$
A \cong B \Longleftrightarrow T^{-1}(A)=T^{-1}(B) \text { and } T^{+}(A)=T^{+}(B) .
$$

It is an equivalence relation which induces a partition $P^{*}(Y)$. An equivalence class of the relation is called a $T$-rough set. Therefore a $T$-rough set of $Y$ is a family of all subsets of $Y$ having the same lower and upper inverse under $T$.

Definition 3.6. Let $R$ and $S$ be two commutative rings and $T: R \rightarrow P^{*}(S)$ be a set-valued mapping. $T$ is called a set-valued homomorphism if 
(i) $T\left(x_{1}+x_{2}\right)=T\left(x_{1}\right)+T\left(x_{2}\right)$;

(ii) $T\left(x_{1} x_{2}\right)=\left\{a b \mid a \in T\left(x_{1}\right), b \in T\left(x_{2}\right)\right\}$;

(iii) $T(-x)=-T(x)$;

for all $x, x_{1}, x_{2} \in R$.

Examples 3.3(v) and (vi) are set-valued homomorphisms. So a ring homomorphism is a special case of a set-valued homomorphism. Let $\theta$ be a complete congruence relation, i.e., $[x]_{\theta}[y]_{\theta}=[x y]_{\theta}$ and $[x]_{\theta}+[y]_{\theta}=[x+y]_{\theta}$ for all $x, y \in R$. Define $T: R \rightarrow P^{*}(R)$ by $T(x)=[x]_{\theta}$, then $T$ is a set-valued homomorphism. Further, the rough sets are $T$-rough sets.

Lemma 3.7. Let $R$ and $S$ be two commutative rings and $T: R \rightarrow P^{*}(S)$ be a setvalued homomorphism. If $U$ is a subring of $S$ and $T^{+}(U), T^{-1}(U)$ are nonempty, then $T^{+}(U)$ and $T^{-1}(U)$ are subrings of $R$.

Proof. Let $x, y \in T^{+}(U)$, by Definition 3.2, $T(x), T(y) \subseteq U$. Since $U$ is a subring of $S$, we have $T(x-y)=T(x)-T(y) \subseteq U$ and $T(x y)=\{a b \mid a \in$ $T(x), b \in T(y)\} \subseteq T(x) T(y) \subseteq U$. It shows that $x-y, x y \in T^{+}(U)$. Moreover, let $x, y \in T^{-1}(U)$, by Definition 3.2, $T(x) \cap U \neq \emptyset$ and $T(y) \cap U \neq \emptyset$. Suppose $a \in T(x) \cap U$ and $b \in T(y) \cap U$. Since $U$ is a subring of $S, a-b \in U$ and $a-b \in T(x)-T(y)=T(x-y)$, thus $a-b \in T(x-y) \cap U$, hence $T(x-y) \cap U \neq \emptyset$. So $x-y \in T^{-1}(U)$. Again, $a b \in\{a b \mid a \in T(x), b \in T(y)\}=T(x y)$ and $a b \in U$. This implies that $T(x y) \cap U \neq \emptyset$. So $x y \in T^{-1}(U)$.

Lemma 3.8. [10] Let $R$ and $S$ be two commutative rings and $T: R \rightarrow P^{*}(S)$ be a set-valued homomorphism. If $A$ is an ideal of $S$ and $T^{+}(A) \neq \emptyset$ and $T^{-1}(A) \neq \emptyset$, then $T^{+}(A), T^{-1}(A)$ are ideals of $R$.

Corollary 3.9. Let $R$ and $S$ be two commutative rings and $T: R \rightarrow P^{*}(S)$ be a set-valued homomorphism. If $A$ is an ideal of $S$ and $T^{-1}(A) \neq \emptyset$ and $T^{+}(A) \neq \emptyset$ then $\left(T^{+}(A), T^{-1}(A)\right)$ is a $T$-rough ideal of $R$.

Recently, we have studied some properties of $T$-rough ideal and $T$-rough prime ideal [10].

Theorem 3.10. Let $R$ and $S$ be two commutative rings and $T: R \rightarrow P^{*}(S)$ be a set-valued homomorphism. If $A$ is a semiprime ideal of $S$ and $T^{+}(A) \neq \emptyset$, then $T^{+}(A)$ is a semiprime ideal of $R$.

Proof. Let $x \in R$ and $x^{2} \in T^{+}(A)$, then $T\left(x^{2}\right) \subseteq A$. But we have $\left\{a^{2} \mid a \in\right.$ $T(x)\} \subseteq\{a b \mid a \in T(x), b \in T(y)\}=T\left(x^{2}\right) \subseteq A$. Then for all $a \in T(x), a^{2} \in A$. Since $A$ is a semiprime ideal of $S$, We have $a \in A$. Hence $T(x) \subseteq A$. It implies that $x \in T^{+}(A)$. Therefore $T^{+}(A)$ is a semiprime ideal of $R$.

Theorem 3.11. Let $R$ and $S$ be two commutative rings and $T: R \rightarrow P^{*}(S)$ be a set-valued homomorphism such that $\left\{a^{2} \mid a \in T(x)\right\}=T\left(x^{2}\right)$. If $A$ is a semiprime ideal of $S$ and $T^{-1}(A) \neq \emptyset$, then $T^{-1}(A)$ is a semiprime ideal of $R$.

Proof. Let $x \in R$ and $x^{2} \in T^{-1}(A)$, then $T\left(x^{2}\right) \cap A \neq \emptyset$. So, there is $b \in T(x)$ such that $a=b^{2} \in A$. Since $A$ is a semiprime ideal of $S$, we have $b \in A$. Hence $b \in T(x) \cap A$. It implies that $T(x) \cap A \neq \emptyset$. Hence $x \in T^{-1}(A)$. Therefore $T^{-1}(A)$ 
is a semiprime ideal of $R$.

Corollary 3.12. Let $R$ and $S$ be two commutative rings and $T: R \rightarrow P^{*}(S)$ be a set-valued homomorphism such that $\left\{a^{2} \mid a \in T(x)\right\}=T\left(x^{2}\right)$. If $A$ is a semiprime ideal of $S$ and $T^{-1}(A) \neq \emptyset$ and $T^{+}(A) \neq \emptyset$, then $\left(T^{+}(A), T^{-1}(A)\right)$ is a $T$-rough semiprime ideal of $R$.

The following examples show that the converse of Lemma 3.8 and Theorem 3.10 do not hold in general.

Example 3.13. Let $\mathbf{Z}$ be integer numbers set and $T: \mathbf{Z} \rightarrow P^{*}(\mathbf{Z})$ where for every $n \in \mathbf{Z}, T(n)=n \mathbf{Z}$. If $A=\{ \pm 1,0, \pm 6, \pm 12, \pm 18, \ldots\}$, then $T^{+}(A)=6 \mathbf{Z}$.

Example 3.14. Suppose $\theta$ be an equivalence relation on $\mathbf{Z}$ by setting

$$
x \theta y \Longleftrightarrow 12 \mid x-y \text {. }
$$

And $T: \mathbf{Z} \rightarrow P^{*}(\mathbf{Z})$ where for every $n \in \mathbf{Z}, T(n)=[n]_{\theta}$. If

$$
A=\{0,6,12, \pm 18, \pm 24, \ldots\}
$$

then $T^{-1}(A)=6 \mathbf{Z}$.

\section{T-ROUGH FUZZY SEMIPRIME IDEAL}

Theory of fuzzy sets initiated by L. A. Zadeh [30]. As a natural need, D. Dubois and H. Prade [5, 6] combined fuzzy sets and rough sets in a fruitful way by defining rough fuzzy sets and fuzzy rough sets. Rough fuzzy sets and fuzzy rough sets are also studied by R. Biswas [1, 2], A. Nakamura [14], S. Nanda [15]. Several research directions have been suggested on fuzzy rough sets and rough fuzzy sets. In this section, we introduce the $T$-rough fuzzy semiprime ideal in a commutative ring and give some properties of such ideals.

Definition 4.1. Let $(U, \theta)$ be an approximation space. A subset fuzzy is a mapping $\mu$ from $U$ to $[0,1]$. If $x \in U$, we define

$$
\underline{\operatorname{Apr}}(\mu)(x)=\bigwedge_{a \in[x]_{\theta}} \mu(a) ; \overline{A p r}(\mu)(x)=\bigvee_{a \in[x]_{\theta}} \mu(a) .
$$

They are called, respectively, the lower and upper approximation of the fuzzy subset $\mu$. $A p r(\mu)=(\operatorname{Apr}(\mu), \overline{A p r}(\mu))$ is called a rough fuzzy set with respect to $\theta$ if $\underline{A p r}(\mu) \neq \overline{A p r}(\mu))$. Let $\mu$ be a subset fuzzy of $U, \lambda \in[0,1]$. Then the sets

$$
\mu_{\lambda}=\{x \in U \mid \mu(x) \geq \lambda\} ; \mu_{\lambda}^{+}=\{x \in U \mid \mu(x)>\lambda\}
$$

are called, respectively, $\lambda$-levelest and $\lambda$-strong levelest of the fuzzy set $\mu$.

Definition 4.2. A fuzzy subset $\mu$ of a ring $R$ is called a fuzzy ideal [11] if

(i) $\mu(x-y) \geq \mu(x) \wedge \mu(y)$ for all $x, y \in R$;

(ii) $\mu(x y) \geq \mu(x) \vee \mu(y)$ for all $x, y \in R$. 
It is a fuzzy prime ideal if $\mu(x y)=\mu(x)$ or $\mu(x y)=\mu(y)$ and it is called a fuzzy semiprime ideal if $\mu\left(x^{2}\right)=\mu(x)$. It is obvious that every fuzzy prime ideal is a fuzzy semiprime ideal but converse of that is not true.

Example 4.3. Let $\mu$ be fuzzy set of $\mathbf{Z}$ by setting

$$
\mu(n)= \begin{cases}1, & \text { if } p q \mid n \\ \alpha, & \text { otherwise }\end{cases}
$$

where $p \neq q$ be a prime integer or zero and $0 \leq \alpha<1$. Then $\mu$ is a semiprime ideal of $\mathbf{Z}$ that is not prime ideal.

Theorem 4.4. Let $\mu$ be a fuzzy subset of a commutative ring $R$. Then $\mu$ is a fuzzy ideal( fuzzy semiprime ideal) of $R$ iff $\mu_{\lambda}, \mu_{\lambda}^{+}$are, if they are nonempty, ideals (semiprime ideals) of $R$ for every $\lambda \in[0,1]$.

Proof. Suppose for every $\lambda \in[0,1], \mu_{\lambda}, \mu_{\lambda}^{+}$are, if they are nonempty, semiprime ideals. By Definition 4.2(ii),$\mu\left(x^{2}\right) \geq \mu(x)$. Now if $\mu\left(x^{2}\right)>\mu(x)$ for some $x \in R$, let $\lambda \in[0,1]$ such that $\mu\left(x^{2}\right)>\lambda>\mu(x)$, then $x^{2} \in \mu_{\lambda}$ but $x \notin \mu_{\lambda}$. It is a contradiction to semiprimness of $\mu_{\lambda}$. Conversely, suppose $\mu\left(x^{2}\right)=\mu(x)$ for all $x \in R$. Let $x^{2} \in \mu_{\lambda}$, then $\mu(x)=\mu\left(x^{2}\right) \geq \lambda$. Hence $x \in \mu_{\lambda}$. For $\mu_{\lambda}^{+}$can be seen in a similar way.

The two following theorems have been proved in [14]:

Theorem 4.5. Let $\theta$ is an equivalence relation on commutative ring $R$ and $\mu$ is a fuzzy ideal of $R$, then $\overline{A p r}(\mu)$ is a fuzzy ideal of $R$.

Theorem 4.6. Let $\theta$ is a complete congruence relation on commutative ring $R$ and $\mu$ is a fuzzy ideal of $R$, then $\operatorname{Apr}(\mu)$ is a fuzzy ideal of $R$ if $\operatorname{Apr}(\mu) \neq \emptyset$.

First of all, we define $T$-rough fuzzy set and $T$-rough fuzzy semiprime ideal.

Definition 4.7. Let $R$ and $S$ be two commutative rings and $T: R \rightarrow P^{*}(S)$ be a set-valued homomorphism. If $\mu$ is a fuzzy ideal of $S$. For every $x \in R$, we define

$$
T^{+}(\mu)(x)=\bigwedge_{a \in T(x)} \mu(a) \quad ; \quad T^{-1}(\mu)(x)=\bigvee_{a \in T(x)} \mu(a) .
$$

$T^{+}(\mu)$ and $T^{-1}(\mu)$ are called, respectively, the lower $T$-rough and the upper $T$ rough fuzzy subsets of $R$ with respect to the fuzzy set of $\mu \cdot\left(T^{+}(\mu), T^{-1}(\mu)\right)$ is said to be $T$-rough fuzzy set of $R$ with respect to the fuzzy set of $\mu$. If $T^{+}(\mu)$ and $T^{-1}(\mu)$ are fuzzy prime(semiprime)ideals, $\left(T^{+}(\mu), T^{-1}(\mu)\right)$ is said to be $T$-rough prime(semiprime) fuzzy ideal of $R$.

Lemma 4.8. Let $R$ and $S$ be two commutative rings and $T: R \rightarrow P^{*}(S)$ be a set-valued homomorphism. If $\mu$ is a fuzzy ideal of $S$, then for all $\lambda \in[0,1]$,

(1) (i) $\left(T^{+}(\mu)\right)_{\lambda}=T^{+}\left(\mu_{\lambda}\right) ; \quad$ (ii) $\left(T^{-1}(\mu)\right)_{\lambda}=T^{-1}\left(\mu_{\lambda}\right)$.

(2) (i) $\left(T^{+}(\mu)\right)_{\lambda}^{+}=T^{+}\left(\mu_{\lambda}^{+}\right) ; \quad$ (ii) $\left(T^{-1}(\mu)\right)_{\lambda}^{+}=T^{-1}\left(\mu_{\lambda}^{+}\right)$. 
Proof. (1-(i)).

$$
\begin{aligned}
x \in\left(T^{+}(\mu)\right)_{\lambda} & \Leftrightarrow T^{+}(\mu)(x) \geq \lambda \Leftrightarrow \bigwedge_{a \in T(x)} \mu(a) \geq \lambda \\
& \Leftrightarrow \text { for all } a \in T(x), \mu(a) \geq \lambda \\
& \Leftrightarrow T(x) \subseteq \mu_{\lambda} \Leftrightarrow x \in T^{+}\left(\mu_{\lambda}\right) .
\end{aligned}
$$

(1-(ii)).

$$
\begin{aligned}
x \in\left(T^{-1}(\mu)\right)_{\lambda} & \Leftrightarrow T^{-1}(\mu)(x) \geq \lambda \Leftrightarrow \bigvee_{a \in T(x)} \mu(a) \geq \lambda \\
& \Leftrightarrow \text { for some } a \in T(x), \mu(a) \geq \lambda \\
& \Leftrightarrow T(x) \cap \mu_{\lambda} \neq \emptyset \Leftrightarrow x \in T^{-1}\left(\mu_{\lambda}\right) .
\end{aligned}
$$

For(2(i), (ii)) can be seen in a similar way.

The following theorems are straightforward.

Theorem 4.9. Let $R$ and $S$ be two commutative rings and $T: R \rightarrow P^{*}(S)$ be a set-valued homomorphism. If $\mu$ is a fuzzy ideal of $S$, then $T^{+}(\mu)$ and $T^{+}(\mu)$ are fuzzy ideals of $R$.

If $\theta$ is a complete congruence relation on $R$ and define $T: R \rightarrow P^{*}(R)$ where $T(x)=[x]_{\theta}$ for every $x \in R$, we obtained Theorems 4.5, 4.6.

Theorem 4.10. If $\mu$ is a fuzzy ideal of $S$ and $T: R \rightarrow P^{*}(S)$ be a set-valued homomorphism. If $\mu$ is a fuzzy semiprime ideal in $S$, then $T^{+}(\mu), T^{-1}(\mu)$ are fuzzy semiprime ideals of $R$.

\section{T-Rough QUOTIENT IDEAL OF RINGS}

Let $R$ and $S$ be two commutative rings and $T: R \rightarrow P^{*}(S)$ be a set-valued homomorphism. Suppose $R / T=\{T(x) \mid x \in R\}$. It is clear that $R / T$ is a commutative ring.

Definition 5.1. Let $R$ and $S$ be two commutative rings and $T: R \rightarrow P^{*}(S)$ be a set-valued homomorphism. The lower $T$-rough quotient and the upper $T$-rough quotient for $A \in P^{*}(S)$ are, respectively,

$$
\left(T^{+}(A)\right) / T=\{T(x) \mid T(x) \subseteq A\} ;\left(T^{-1}(A)\right) / T=\{T(x) \mid T(x) \cap A \neq \emptyset\} .
$$

Lemma 5.2. Let $R$ and $S$ be two commutative rings and $T: R \rightarrow P^{*}(S)$ be a set-valued homomorphism. If $A \in P^{*}(S)$ be an ideal of $S$ and $\left(T^{+}(A)\right) / T \neq \emptyset$, then $\left(T^{+}(A)\right) / T$ is an ideal of $R / T$.

Proof. Suppose $T(x), T(y) \in\left(T^{+}(A)\right) / T$. Since $A \in P^{*}(S)$ is an ideal of $S$, So $T(x-y)=T(x)-T(y) \subseteq A$. Then $T(x)-T(y) \in\left(T^{+}(A)\right) / T$. Now suppose

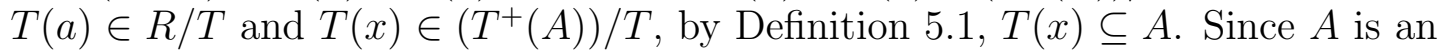
ideal of $S$, thus $T(x a) \subseteq T(x) T(a) \subseteq A$, therefore $T(x a) \in\left(T^{+}(A)\right) / T$. 
Lemma 5.3. Let $R$ and $S$ be two commutative rings and $T: R \rightarrow P^{*}(S)$ be a setvalued homomorphism such that $\{a b \mid a \in T(x), b \in T(y)\}=T(x y)=T(x) T(y)$. If $A \in P^{*}(S)$ be an ideal of $S$ and $\left(T^{-1}(A)\right) / T \neq \emptyset$, then $\left(T^{-1}(A)\right) / T$ is an ideal of $R / T$.

Proof. Suppose $T(x), T(y) \in\left(T^{-1}(A)\right) / T$. Thus $T(x) \cap A \neq \emptyset \neq T(y) \cap A$. Hence, there exist $a \in T(x) \cap A$ and $b \in T(y) \cap A$. Since $A \in P^{*}(S)$ is an ideal of $\mathrm{S}$, So $a-b \in T(x)-T(y)=T(x-y), a-b \in A$. Therefore $a-b \in T(x-y) \cap A$. Then $T(x)-T(y) \in\left(T^{-1}(A)\right) / T$. Now suppose $T(a) \in R / T$ and $T(x) \in\left(T^{-1}(A)\right) / T$, by Definition 5.1, $T(x) \cap A \neq \emptyset$. There exists $b \in T(x) \cap A$. Since $A$ is an ideal, $T(a) b \subseteq A$ and $T(a) b \subseteq T(x a)=T(x) T(a)$. Then $T(a) b \subseteq T(x a) \cap A$. Hence $T(x) T(a)=T(x a) \in\left(T^{-1}(A)\right) / T$.

Definition 5.4. Let $R$ and $S$ be two commutative rings and $T: R \rightarrow P^{*}(S)$ be a set-valued mapping. $T$ is called a strong set-valued homomorphism if

(i) $T\left(x_{1}+x_{2}\right)=T\left(x_{1}\right)+T\left(x_{2}\right)$;

(ii) $T\left(x_{1} x_{2}\right)=\left\{a b \mid a \in T\left(x_{1}\right), b \in T\left(x_{2}\right)\right\}=T\left(x_{1}\right) T\left(x_{2}\right)$;

(iii) $\left\{a^{2} \mid a \in T(x)\right\}=T\left(x^{2}\right)$;

(iv) $T(-x)=-T(x)$;

for all $x, x_{1}, x_{2} \in R$.

We can obtain the following propositions by direction from the definition.

Proposition 5.5. Let $R$ and $S$ be two commutative rings and $T: R \rightarrow P^{*}(S)$ be a strong set-valued homomorphism. If $A \subseteq S$ be a semiprime ideal of $S$ and $\left(T^{+}(A)\right) / T \neq \emptyset$, then $\left(T^{+}(A)\right) / T$ is a semiprime ideal of $R / T$.

Proposition 5.6. Let $R$ and $S$ be two commutative rings and $T: R \rightarrow P^{*}(S)$ be a strong set-valued homomorphism. If $A \subseteq S$ be a semiprime ideal of $S$ and $\left(T^{-1}(A)\right) / T \neq \emptyset$, then $\left(T^{-1}(A)\right) / T$ is a semiprime ideal of $R / T$.

Corollary 5.7. Let $\theta$ is a complete congruence relation on commutative ring on $S$ and $A$ an ideal of $S$, then $(\underline{A p r}(A)) / \theta=\left\{[x]_{\theta} \mid[x]_{\theta} \subseteq A\right\}$ and $(\overline{\operatorname{Apr}}(A)) / \theta=$ $\left\{[x]_{\theta} \mid[x]_{\theta} \cap A \neq \emptyset\right\}$ are ideals of $S / \theta$.

Corollary 5.8. Let $\theta$ is a complete congruence relation on commutative ring on $S$ and $A$ is a semiprime ideal of $S$, then $(A p r(A)) / \theta$ and $(\overline{A p r}(A)) / \theta$ are rough quotient semiprime ideals.

For proving the two above corollaries, it is enough we define $T: S \rightarrow P^{*}(S)$ where $T(x)=[x]_{\theta}$ for all $x \in S$.

\section{Conclusion}

The rough sets theory is regarded as a generalization of the classical sets theory. A key notion in rough set is an equivalence which is sometime difficult to be obtained in rearward problems due to vagueness and incompleteness of human knowledge. In the present paper, we substituted a universe set by a ring 
and introduced the set-valued homomorphism and the strong set-valued homomorphism, $T$-rough semiprime ideals and $T$-rough fuzzy semiprime ideals in a commutative ring based on definitions in [5]. We discussed the relations between upper(lower) $T$ - rough semiprime ideals and upper (lower) approximations of their homomorphism images. We extended some theorems which have been proved $[5$, $6,12]$. In addition, we introduced the notion of lower and upper $T$-rough quotient set in a ring and investigated some their interesting properties.

Acknowledgment The author is highly grateful to Professor S. Jafari of Department of Mathematics College of Vestsjaelland South for his valuable comments and suggestions for improving paper.

\section{REFERENCES}

1. R. Biswas, On rough sets and fuzzy rough sets , Bull. Pol.Acad. Sci. Math. 42 (1994), $345-349$.

2. R. Biswas, On rough fuzzy sets, Bull. Pol. Acad. Sci. Math.42 (1994), 352-355.

3. R. Biswas, S. Nanda, Rough groups and rough subgroups, Bull. Polish Acad. Sci. Math 42(1994), 251-254.

4. Z. Bonikowaski, Algebraic structures of rough sets, in: W.P. Ziarko (Ed.), Rough Sets, Fuzzy Sets and Knowledge Discovery, Springer-Verlag, Berlin, 1995, pp. 242-247.

5. B. Davvaz, A short note on algebraic T-rough sets, Information Sciences 178 (2008), 32473252 .

6. B. Davvaz, Roughness in rings, Inform. Sci. 164 (2004), 147-163.

7. D. Dubois, H. Prade, Rough fuzzy sets and fuzzy rough sets, Int. J. General Syst. 17 (2-3)(1990), 191-209.

8. D. Dubois, H. Prade, Two fold fuzzy sets and rough sets-some issues in knowledge representation, Fuzzy Sets Syst. 23 (1987), 3-18.

9. S. B. Hosseini, N. Jafarzadeh, A. Gholami, T-rough Ideal and T-rough Fuzzy Ideal in a Semigroup , Advanced Materials Research Vols. 433-440 (2012) pp 4915-4919.

10. S. B. Hosseini, N. Jafarzadeh, A. Gholami, Some Results on T-rough (prime, primary) Ideal and T-rough Fuzzy (prime, primary) Ideal on Commutative Rings, Int. J. Contemp. Math. Sciences, Vol. 7, 2012, no. 7, 337-350

11. T. Iwinski, Algebraic approach to rough sets, Bull. PolishAcad. Sci. Math. 35 (1987), 673683.

12. Osman Kazanci, Sultan Yamak, B. Davvaz, On the structure of rough prime (primary) ideals and rough fuzzy prime (primary) ideals in commutative rings, Information Sciences, 178 (2008), 2349-2359.

13. N. Kuroki, Rough ideals in semigroups, Inform. Sci. 100 (1997), 139-163.

14. A. Nakamura, Fuzzy rough sets, Note on Multiple-valued Logic in Japan, 9 (8) (1988), 1-8.

15. S. Nanda, Fuzzy rough sets, Fuzzy Sets and Systems, 45 (1992), 157-160.

16. Z. Pawlak, Rough sets basic notions, ICS PAS Rep. 436 (1981).

17. Z. Pawlak, Rough sets, Int. J. Inform. Comput. Sci. 11 (1982), 341-356.

18. Z. Pawlak, Rough sets power set hierarchy, ICS PAS Rep.470 (1982).

19. Z. Pawlak, Rough sets algebraic and topological approach, ICS PAS Rep. 482 (1982).

20. Z.Pawlak, Rough sets and fuzzy sets, Fuzzy Sets and Systems17 (1985), 99-102.

21. Z. Pawlak, Rough sets, Theoretical Aspects of Reasoning about Data, Kluwer Academic Publishers, Dordrecht, 1991.

22. Z. Pawlak, Rough Sets - Theoretical Aspects of Reasoning about Data, Kluwer Academic Publishing, Dordrecht, 1991.

23. Z. Pawlak, Some remarks on rough sets, Bull. Pol. Acad.Tech. 33 (1985). 
24. Z. Pawlak, A. Skowron, Rough sets and Boolean reasoning, Information Sciences, 177 (2007), 41-73.

25. Z. Pawlak, A. Skowron, Rough sets: some extensions, Information Sciences, 177 (2007), 28-40.

26. Z. Pawlak, A. Skowron, Rudiments of rough sets, Information Sciences, 177 (2007), 3-27.

27. A. Rosenfeild, Fuzzy Groups ,Journal of Mathematical Analysis and Appl ication , 35, (1971) 512-517.

28. Qi-Mei Xiao, Zhen-Liang Zhang, Rough prime ideals and rough fuzzy prime ideals in semigroups, Information Sciences, 176 (2006), 725-733.

29. S. Yamak, O. Kazanci, B. Davvaz, Generalized lower and upper approximations in a ring ,Information Sciences 180 (2010) 1759-1768.

30. L.A. Zadeh, Fuzzy sets, Inform. Control 8 (1965) 338-353.

31. W. Zhang, W. Wu, Theory and Method of Roughness, Science Press, Beijing, 2001.

1, Department of Mathematics, Sari Branch, Islamic Azad University, Sari, IRAN.

E-mail address: sbhosseini@iausari.ac.ir 